\title{
Fake News and Disinformation in Health Care- Challenges and Technology Tools
}

\author{
Maria TSIRINTANI ${ }^{1}$ \\ University of West Attica, Athens, Greece
}

\begin{abstract}
The aim of this study is to define and analyze the phenomenon of fake news and disinformation from social media in healthcare ecosystem. Social media and web platforms can be used to spread misinformation, which produces harmful consequences to global health and well-being. A qualitative research was carried out by using semi structured interview with experts for collecting the data investigating the social, legal and technical environment that promote this phenomenon. In order to shed some light, software tools and web platforms are suggested to those in charge of educating users on their best use of internet for this purpose.
\end{abstract}

Keywords. Healthcare, Social Media, Fake news, Disinformation

\section{Introduction}

The Internet and the Social media platforms have taken a leading role in the way we obtain health information for our decisions. However, given the large amount of inaccurate information online, people can easily become misinformed [1]. The impact of Fake news is a major concern in public health as it can reduce or increase the effectiveness of programs, campaigns and initiatives aimed at citizens' health, awareness and well-being [2].

Fake news concern false, inaccurate or misleading information designed, presented or promoted to internationally cause public harm or for profit. Disinformation is not always fake but it is fabricated information blended with facts [3]. The most recent topic fueling disinformation is the pandemia of Covid-19 [4]. There is a need to define and analyze the challenges of this phenomenon and present the existing tools of Informatics to educate and reduce the negative consequences in health care and public health.

In this study, we are investigating the social, legal and technical environment that promote inaccurate health information and we are presenting existing software tools and web platforms to identify, evaluate or educate the users especially educators.

\section{Methods}

The methodology for conducting qualitative research was based on the six-step development model in order to extract as relevant and safe conclusions as possible [5].

\footnotetext{
${ }^{1}$ Corresponding Author, Maria TSIRINTANI, Professor, Health Information Systems, Health and Social Care Management Sector, Department of Business Administration, Faculty of Business, Economics and Social Sciences, University of West Attica, E-mail: martsi@uniwa.gr.
} 
The sample method chosen for the collection of the data was the semi-structured interview [6,7]. An interview with seven experts (three of them in social media, two legal and two web platform developers in healthcare) in Athens, Greece took place on September 2020. The questions that composed the interview questionnaire were structured into five modules: A) the demographic information of respondents, B) the legal environment for social media C) the social impact for fake news and disinformation, D) web platforms and existing education software tools to identify, evaluate or educate about fake news and disinformation, and E) the attempt to learn the personal view of the respondents about the challenges for healthcare and public health.

Data Analysis was done by encoding the questions of the interview. The qualitative research encountered difficulties such as, lack of previous similar research to present several aspects of the phenomenon and the contrast of principles and rights of users on Internet with the current legislation in healthcare.

\section{Results and Discussion}

According the data analysis from the expert's interview from B and C module: Society has been given enormous importance to information as business resource after the digital revolution and new technologies. In all scientific fields not only in healthcare, there is a great need to effectively curb misinformation. There is an interconnection of the most fundamental infrastructures of vital importance for e-governance such as e-health with increasing their complexity. Interoperability and digital convergence of even devices via the Internet of Things, Cloud Computing, and development of Artificial Intelligence technologies have augmented the interest for new investments such as smart houses and telemedicine.

There is a rupture with traditional ways of information (younger ages, developing countries, critical economic and political periods). Moreover, we have new features of cybercrime due to easier access to technology anonymity (concealment of identity) of the perpetrators (such as dark Web and proxy). It is necessary to provide the cross-border nature of crime - problems of jurisdiction and cooperation of states [8]. International consensus is needed on what criminal behavior means for Internet, globalization of crime against existing legislation, invisible crime, wider victimization dimensions, minimum cost of committing a crime with low risk of finding multiple benefits (cost benefit analysis, rational choice theories).

Internet misinformation has a qualitative change / change of context and quantitative differences / intense differences (use of bots in spreading false news). For managerial and legislation purpose there is conflict for the issue of categorization between a genuine cybercrime or traditional crime with new ways of doing it. Strict criminal treatment is considered a solution but there is contrast of principles and rights of users on Internet and legislation in different countries.

According the data analysis from the expert's interview from $\mathrm{D}$ and $\mathrm{E}$ module: Society False news in healthcare is not easy to spot and manage [9,10]. Prevention is mostly occasional. Mostly there is occasional utilization of readers with reports and crowdsourcing in healthcare and public health. Social media platforms must fundamentally rethink their products to reduce the health risks posed by disinformation and misinformation $[11,12]$

Nowadays, there is a variety of platforms and information systems that create fake news and spread it easily and quickly (software that composes texts on its own by taking 
information by the Internet and also using Artificial Intelligence). There are more than eighty software tools to suggest in specific categories of using:

1. Investigate Bot/spam, tools for automatically detecting and identifying unreliable accounts that may spread false news / misinformation such as "Botometer" an online machine learning program that classifies twitter accounts as bot or human, (https://botometer.iuni.iu.edu/),

2. Credibility scoring, platforms and tools that evaluate and provide an indicator of reliability in articles and websites distinguishing between high and low quality information (https://fakerfact.org/about),

3. Misinformation tracking tools, such as Hoaxy online tool that depicts the spread of articles on the internet, checks facts from 2016, calculates a bot rating as a measure of the likelihood that an account will be bot on twitter (Provided by Indiana University Social Networking)

4. Artificial Intelligence and Machine Learning Tools. The US National Science Foundation supports "Claimbuster" which uses national language editing to highlight real claims in texts techniques (https://idir.uta.edu/claimbuster/),

5. Bot sentinel, free platform for locating and tracking trollbots in twitter accounts. Uses machine learning to study accounts to classify them as trustworthy and locate bots (https://botsentinel.com/),

6. Online verification tools, simplify the verification or refutation of a news item or video (https://captainfact.io/),

7. Whitelisting, tools that block misleading ads or websites (https://adblockplus.org),

8. Codes and standards, methods and initiatives of experts, especially for journalists, certification of publishers and creation of methods for verifying information before fact checking news, (https://credibilitycoalition.org/),

9. Education/training, web-based education tools designed to improve media literacy (https://fakey.iuni.iu.edu/).

\section{Conclusions}

After the qualitative research, it was found that fake news and disinformation detection is partially automated and social media companies need to invest more in order to reduce the negative consequences. False news in healthcare is not easy to spot and manage. Prevention is mostly occasional in healthcare and public health. Strict criminal treatment is considered a solution (an obstacle if it is the result of criminal populism).

Future research will be necessary to define more aspects and challenges for fake news and disinformation, such as changes in the legislative framework in terms of the effects that fake news have on businesses and states in the decision-making process. So far, the consequences of these decisions have not been adequately accounted for in legal and social terms between states. For the technical approach, evaluating the existing software tools of the Web to educate about, prevent or distinguish this phenomenon, will be valuable for the users and web software developers. 


\section{Acknowledgments}

This study has been done within the framework of the Postgraduate Health and Social Care Management Program of the University of West Attica.

\section{References}

[1] Thompson B, Laser D. Public Health and Online Misinformation. Ann. Rev. Public Health 2020;(41): 433- 451.

[2] Pulido C. A New Application of Social Impact in Social Media for Overcoming Fake News in Healthcare, Int. J. Environ. Res. Public Health 2020 Apr;17(7):2430.

[3] Final report of the High Level Expert Group of Fake News and Online Disinformation. Available at: https://ec.europa.eu/digital-single-market/en/news/final-report-high-level-expert-group-fake-news-andonline-disinformation

[4] Brannen S, Simon F, Howard P, Nielsen R. Types, sources, and Claims of COVID-19 Misinformation: Reuters Institute 2020. Available at: http://www.primaonline.it/wp-content/uploads/2020/04/COVID19_reuters.pdf

[5] Mantzoukas S. Qualitative research in six easy steps, Epistemology, methods and presentation, Nosileftiki 2007;(46):88-98

[6] The Sage encyclopedia of qualitative research methods, Given LM edit. SAGE Publ. Inc; 2008

[7] Giacomini M, Cook D. Users guides to the medical literature: XXIII. Qualitative research in health care: Are the results of the study valid? J Am Med Assoc. 2000;(284):357-362

[8] Mitrosili M. Health Law: Papazisis Publications; 2009

[9] Goula A. Organizational Culture of Health Services: Papazisis Publications; 2014

[10] Treharne T, Papanikitas A. Defining and detecting fake news in health and medicine reporting. J Royal Soc. Medic. 2020;(113):302-305

[11] Simpson E, Conner A. Fighting coronavirus misinformation and Disinformation. Preventive Product Recommendations for Social Media Platforms: Center for American Progress. Available at: https://www.americanprogress.org/issues/technology-policy/reports/2020/08/18/488714/fightingcoronavirus-misinformation-disinformation/

[12] Tackling online disinformation, Shaping Europe's digital future. Available at: https://digitalstrategy.ec.europa.eu/en/policies/online-disinformation 